Ralf Tunn, Engelbert Hanzal, Daniele Perucchini (Hrsg.)

Urogynäkologie in Praxis und Klinik 



\section{Urogynäkologie in Praxis und Klinik}

3. Auflage

Herausgegeben von

Ralf Tunn, Engelbert Hanzal und Daniele Perucchini

\section{DE GRUYTER}


Herausgeber

Prof. Dr. med. Ralf Tunn

St. Hedwig Kliniken

Klinik für Urogynäkologie

Große Hamburger Str. 5-11

10115 Berlin

Email: r.tunn@alexianer.de

ao. Univ. Prof. Dr. med. Engelbert Hanzal

Kontinenz- und Beckenbodenzentrum

Medizinische Universität Wien

Universitätsklinik für Frauenheilkunde

Währinger Gürtel 18-20

1090 Wien

Österreich

Email: engelbert.hanzal@meduniwien.ac.at
Priv.-Doz. Dr. med. Daniele Perucchini

Blasenzentrum AG, Zürich

Gottfried-Keller-Strasse 7

8001 Zürich

Schweiz

Email: perucchini@blasenzentrum.ch

und

Klinik für Gynäkologie

Universitätsspital Zürich

8091 Zürich

ISBN: 978-3-11-065434-9

e-ISBN (PDF): 978-3-11-065790-6

e-ISBN (EPUB): 978-3-11-065447-9

Library of Congress Control Number: 2021934663

\section{Bibliografische Information der Deutschen Nationalbibliothek}

Die Deutsche Nationalbibliothek verzeichnet diese Publikation in der Deutschen Nationalbibliographie; detaillierte bibliografische Daten sind im Internet über http://dnb.d-nb.de abrufbar.

Der Verlag hat für die Wiedergabe aller in diesem Buch enthaltenen Informationen mit den Autoren große Mühe darauf verwandt, diese Angaben genau entsprechend dem Wissensstand bei Fertigstellung des Werkes abzudrucken. Trotz sorgfältiger Manuskriptherstellung und Korrektur des Satzes können Fehler nicht ganz ausgeschlossen werden. Autoren und Verlag übernehmen infolgedessen keine Verantwortung und keine daraus folgende oder sonstige Haftung, die auf irgendeine Art aus der Benutzung der in dem Werk enthaltenen Informationen oder Teilen davon entsteht.

Die Wiedergabe der Gebrauchsnamen, Handelsnamen, Warenbezeichnungen und dergleichen in diesem Buch berechtigt nicht zu der Annahme, dass solche Namen ohne weiteres von jedermann benutzt werden dürfen. Vielmehr handelt es sich häufig um gesetzlich geschützte, eingetragene Warenzeichen, auch wenn sie nicht eigens als solche gekennzeichnet sind.

(C) 2021 Walter de Gruyter GmbH, Berlin/Boston

Einbandabbildung: Talaj / iStock / Getty Images Plus

Satz/Datenkonvertierung: L42 AG, Berlin

Druck und Bindung: CPI books GmbH, Leck

www.degruyter.com 